In Crescendo, 2019; 10(1): 223-243

Fecha de recepción: 15 de enero del 2019

Fecha de aceptación: 25 de marzo del 2019

\title{
DIAGNÓSTICO SOBRE LA PROSPECTIVA DE GENERACIÓN DE EMPRESAS Y PROYECTOS PRODUCTIVOS DE EMPRENDIMIENTO SOCIAL Y ECONOMÍAS CREATIVAS EN EL SECTOR SOLIDARIO, PROPUESTOS POR ESTUDIANTES DE LA UCC SEDE MONTERÍA EN EL AÑO 2017
}

DIAGNOSIS ON THE PROSPECTIVE OF GENERATION OF COMPANIES AND PRODUCTIVE PROJECTS OF SOCIAL ENTREPRENEURSHIP AND CREATIVE ECONOMIES IN THE SOLIDARITY SECTOR, PROPOSED BY STUDENTS OF THE UCC SEDE MONTERIA

IN THE YEAR 2017

Celene M. Romero' ${ }^{1}$ Katia I. De La Puente ${ }^{2}$, Luis F. Alfonso ${ }^{3}$

\section{RESUMEN}

La economía solidaria es clave en el proceso de paz en Colombia; Zuluaga, C (2016). Córdoba, posterior a la desmovilización de las (AUC) en 2005, se ha mantenido en el conflicto social y armado, existen extorsiones, desapariciones, homicidios y desplazamientos, además economías ilegales. CINEP (2016). Por lo anterior, El objetivo es diagnosticar la prospectiva que tienen los estudiantes de la UCC Montería, en cuanto al desarrollo de empresas de emprendimiento social y economías creativas, identificar las organizaciones públicas y privadas que apo-

1 Universidad Cooperativa de Colombia. MBA, Especialista en R.H y Docencia, Docente; celene.romeroh@campusucc.edu.co

2 Universidad Cooperativa de Colombia. MBA, Especialista en Marketing, Docente; katia.delapuente@campusucc.edu.co

3 Universidad Cooperativa de Colombia.MBA, Docente; luis.alfonsog@ucc.edu.co 
yen la generación de estas empresas y proponer estrategias de creación por parte de los estudiantes que ofrezcan una solución innovadora a problemas de desarrollo social y económico de forma sostenible, para el post conflicto en la región cordobesa y en el país. El tipo de estudio es descriptivo-cuantitativo e investigación exploratoria; la población total de los estudiantes de la sede UCC sede Montería al año 2017 es 4.090 estudiantes según el DARC (2017); distribuidos en las facultades de Derecho, Ingeniería de Sistemas, Psicología y la Facultad de Ciencias Administrativas, Contables y Afines; de la cual se tomó una muestra de 350 estudiantes utilizando la fórmula para estimar la muestra en poblaciones finitas, con un nivel de confianza de 95\% y una estimación de error del 5\%, se llegó a las siguientes conclusiones: Estudiantes de Derecho y Psicología, tienen menos interés a la hora de emprender, en toda la UCC se imparten asignaturas enfocadas en promover el emprendimiento solidario, pese a esto más del 70\% no tiene un proyecto en mente y los que sí, no contempla el sector social como opción, en Ingeniería de sistemas es donde prima el deseo de solucionar un problema como motivación para emprender y los estudiantes ven como principales limitantes la falta de recursos y la falta de motivación.

PALABRAS CLAVE: Empresas, emprendimiento social, economía, sector solidario.

\begin{abstract}
The solidarity economy is key in the peace process in Colombia; Zuluaga, C (2016). Córdoba, after the demobilization of the (AUC) in 2005, has remained in the social and armed conflict, extortions, disappearances, homicides and displacements, as well as illegal economies. CINEP (2016). Therefore, the objective is to diagnose the prospective students of UCC Monteria, in terms of the development of social entrepreneurship and creative economies, identify public and private organizations that support the generation of these companies and propose creative strategies by students who offer an innovative solution to problems of social and economic development in a sustainable way, for post-conflict in the region of Cordoba and in the country.
\end{abstract}

The type of study is descriptive-quantitative and exploratory research; Through a survey information was collected and the following partial conclusions were reached: Law and Psychology students, have less interest when undertaking, as opposed to the Faculty of Administrative, Accounting and related Sciences, throughout the UCC they are taught subjects focused on promoting solidary entrepreneurship, more than $70 \%$ do not have a project in mind and those that do, does not contemplate the social sector as an option, in systems engineering is where the desire to solve a problem as motivation to undertake and the Students see lack of resources and lack of motivation as the main constraints.

KEY WORDS: Company, solidarity sector, social entrepreneurship, orange economy, cultural industry. 


\section{INTRODUCCIÓN}

Como lo afirma Arana, A (2015); en el posconflicto las cooperativas tienen una gran oportunidad de aportarle al país su modelo de empresa, que podría funcionar en todos los sectores de la economía.

El Departamento de Córdoba, posterior a la desmovilización de las (AUC) en 2005, se ha mantenido en el conflicto social y armado, con extorsiones, desapariciones, homicidios y desplazamientos, además economías ilegales. CINEP (2016). Por esta razón es pertinente realizar este proyecto, ya que es de suma importancia empezar a generar conciencia de que todos debemos aportar a la construcción de la paz y desde la academia es necesario generar proyectos productivos, incentivar a los jóvenes a ser creadores de oportunidades y no depender de un empleo, y la mirada está puesta en el sector solidario, pues no solo en el marco de un postconflicto es viable hablar del modelo solidario, sino que siempre ha sido una de las mejores opciones para generar progreso y dinamizar la economía del país, con empresas sin ánimo de lucro con gran sentido social.

Existe un amplio consenso entre muchos actores, incluidas las Naciones Unidas, la Organización Internacional del Trabajo (OIT) y la Alianza Cooperativa Internacional (ACI), acerca de las empresas del sector solidario; estos afirman que:

"La empresa cooperativa es el tipo de organización más idónea para abordar todas las dimensiones de la reducción de la pobreza y de la exclusión. Es importante el modo en que las cooperativas contribuyen a reducir la pobreza: encuentran oportunidades económicas para sus socios; empoderan a las personas desfavorecidas para que defiendan sus intereses; dan seguridad a los pobres permitiéndoles transformar sus riesgos individuales en riesgos colectivos; y median para que sus socios accedan a los activos que utilizan para ganarse la vida". OIT \& COOP (2016).

En Colombia a junio 30 de 2016, se cuenta que en el registro único empresarial y social (RUES), tenemos 190.467 organizaciones solidarias con matrícula activa y 66.095 con la matrícula renovada y de las entidades con matrícula renovada tenemos que el $12 \%$ son organizaciones de economía solidaria y el $88 \%$ organizaciones solidarias de desarrollo, una cifra bastante alta y que nos sirve de precedente para entender que el país está apostando al emprendimiento social. 
Por su parte la UCC tiene como misión, educar personas con las competencias para responder a las dinámicas del mundo, contribuir a la construcción y difusión del conocimiento, apoyar el desarrollo competitivo del país a través de sus organizaciones y buscar el mejoramiento de la calidad de vida de las comunidades, influidos por la economía solidaria el cual es su ADN y contando que en la actualidad la sede Montería cuenta con 4.090 estudiantes según (DARC, 2017) y que el sector solidario se proyecta como el mayor aportante a la consolidación de la paz en nuestro país por ser el sector con mayor inclusión social; es importante enfocar a los estudiantes a mirar el sector solidario como base de su proyecto de vida; además de apuntar a la creación de industrias culturales y de economías creativas (ICC) como alternativa de desarrollo; teniendo como referencia que tanto la UNESCO como la Unión Europea reconocen que las ICC son un cauce de expresión de la identidad cultural y un instrumento de mediación de la diversidad cultural, aprovechando su potencial para contribuir al desarrollo de la economía. Pallares, S (2011).

Entre tanto, la universidad imparte las cátedras de oportunidades de negocios, plan de negocios, economía solidaria y emprendimiento solidario, en los cuales se ayuda a desarrollar proyectos que son expuestos en ferias empresariales internas realizadas en la sede Montería y a través del premio Rymel Serrano en donde se premia las ideas emprendedoras de los estudiantes de la universidad enfocados al sector solidario y que por ende pueden generar un banco de posibles proyectos productivos de emprendimiento enfocados al sector social; es por esto que se realiza la siguiente formulación del problema: ¿Cuál es la Prospectiva de generación de empresas y proyectos productivos de emprendimiento social y economías creativas en el sector solidario, propuestos por estudiantes de la UCC, Sede Montería?

El objetivo general del proyecto es identificar la prospectiva de generación de empresas y proyectos productivos de emprendimiento social y economías creativas en el sector solidario, liderados por estudiantes de la UCC, Sede Montería; y los objetivos específicos: Diagnosticar cuál es la prospectiva que tienen los estudiantes de la UCC sede Montería, en cuanto al desarrollo de empresas de emprendimiento social y economías creativas, durante el año 2017; identificar las organizaciones públicas y privadas que apoyan la generación de este tipo de empresas, a partir de alianzas estratégicas y proponer estrategias para la creación de este tipo de empresas por parte de los estudiantes de la sede UCC Montería, que ofrezcan una solución innovadora a un problema de desarrollo social y eco- 
nómico de forma sostenible, teniendo en cuenta el contexto socio-económico y cultural de la región.

Como antecedentes tenemos desde lo internacional; investigaciones realizadas en Cuba sobre el emprendimiento de los nativos en el sector turístico; titulado: "Emprendimiento: perspectiva cubana en la creación de empresas familiares" donde los autores analizan las características de los emprendimientos del sector no estatal cubano en el servicio de alojamiento turístico y sus efectos sobre las valoraciones de los clientes internacionales; específicamente en el caso de Cuba, el concepto de emprendedor no ha sido abordado, ya que las formas económicas vigentes eran solamente estatales o de cooperación mixta. Silveira, Y; Cabeza, D \& Fernández, V (2016). Este artículo amplía el marco conceptual explicando el término de emprendimiento turístico, además nos ejemplifica la forma como nuestros estudiantes pueden incursionar en este tipo de emprendimiento en nuestro entorno aprovechando que actualmente Montería y la región cordobesa en general se han convertido en atractivo turístico para nacionales y extranjeros; debido a la diversidad cultural, flora, fauna y las fuentes hídricas como los mares y nuestros ríos.

Otro referente es el artículo titulado "El debate de la creatividad y la economía en las ciudades actuales y el papel de los diferentes actores: Algunas evidencias a partir del caso de estudio de Madrid"; donde se analiza de manera crítica los conceptos de economía creativa y clase creativa a través de una revisión de literatura internacional. De esta forma se ponen de manifiesto las lagunas teóricas y conceptuales de los enfoques dominantes al respecto y propone algunas ideas para interpretar el papel de la creatividad en la economía de las ciudades desde una perspectiva más compleja, y lleva a cabo una aproximación al caso de Madrid. (Prada, 2015). El artículo se toma como referencia ya que sirve para ampliar el marco teórico sobre el tema de la economía cultural y creativa.

Además se toma como referencia el informe realizado por el Programa de las Naciones Unidas para el Desarrollo (PNUD) y las Naciones Unidas para la Educación, la Ciencia y la Cultura (UNESCO), sobre la economía creativa en 2013; que hizo contribuciones significativas al esfuerzo conjunto y constante de expertos de todo el mundo que sostenían que existía un sector productivo valioso, fuerte y considerable; "Estos expertos han proporcionado pruebas concluyentes que demuestran las vías en las que la vanguardia del crecimiento e innovación en la economía contemporánea está formada por sectores, tales como la industria de alta tecnología, la fabricación neo-artesanal, los servicios financieros y empresa- 
riales, las industrias de productos culturales (Incluyendo los medios de comunicación) y demás". Asimismo, mostraron que la inversión en los sectores culturales y creativos puede ser una poderosa opción para el desarrollo, aportando pruebas de que, a pesar de la grave recesión que se ha dado recientemente en el mundo desarrollado, la economía creativa continuaba creciendo en todas partes y, generalmente, más rápidamente que otros sectores, sobre todo en los países del sur global. PNUD \& UNESCO (2013).

En Colombia, se tiene la investigación realizada por Fredy de Jesús Mojica González (2015) sobre las empresas creadas por los egresados entre el año 2010 y 2014 de la Universidad Nacional Abierta y a Distancia UNAD, pertenecientes al Centro de Educación a Distancia CEAD Sogamoso, y que tuvo como producto el capítulo VIII del libro "Retos y Desafíos de la Prospectiva del Emprendimiento Social y Solidario de la ciudades del futuro"; en este documento, se determinaron las principales características de las micro, pequeñas y medianas empresas creadas por los egresados entre el año 2010 y 2014 de la Universidad UNAD de Sogamoso, a través de la aplicación de una encuesta que permitió dar respuesta a la pregunta formulada: ¿Cuáles son las características de las pymes creadas por los egresados Unadistas del CEAD Sogamoso, entre el año 2010 y 2014?; aquí se determinaron características tales como: Actividad de la empresa, mercado objetivo, tamaño de la empresa, tipo de empresa, estructura de la empresa, número de empleados, fuentes de financiación, nivel de formación de los empleados, condiciones laborales, innovación e implementación de tecnología, incorporación de sistemas de calidad, agremiaciones, pertinencia de los conocimientos adquiridos, entre otros aspectos; que nos pueden servir para caracterizar las empresas de nuestros estudiantes, en el caso de encontrar empresas creadas por ellos en el sector social a través de la investigación.

Otra referencia encontrada presenta los resultados de la investigación "Elementos para la construcción del direccionamiento estratégico de Organizaciones Solidarias en Colombia durante el periodo 2014-2018: Una identificación desde el enfoque prospectivo". El desarrollo de esta investigación apuntó desde un enfoque prospectivo, a construir los elementos fundamentales para el direccionamiento estratégico de Organizaciones Solidarias, con los cuales se posibilite la incorporación transversal de la economía solidaria en los distintos temas e instancias del gobierno en curso. Aguilar, C (2014); es de suma importancia este antecedente para la realización de este proyecto ya que aporta datos concretos sobre el estado del sector solidario en Colombia. 
Entre tanto, el estudio realizado por Adeline Nicole Fournier de la universidad central en 2011 sobre el tema de emprendimiento social en ocho de las principales universidades del país (privadas y públicas) reconocidas por sus trabajos en el campo de la administración: Universidad de los Andes, Universidad Externado de Colombia, Universidad del Rosario, Pontificia Universidad Javeriana, Universidad de la Sabana, EAFIT, Universidad Nacional y Universidad EAN; explica que el tema de emprendimiento social implica cierta interdisciplinaridad, la autora analizó si existían programas que abarcaran el tema de emprendimiento social desde las perspectivas de las ciencias sociales y humanas, este es uno de los pocos estudios que se tiene en el país con respecto al tema; dentro de las conclusiones que arrojó esta investigación la autora afirma que la RSE viene siendo abordado por casi todas las universidades estudiadas, bien sea mediante cursos integrados a las mallas de pregrado y posgrado, o de forma más profunda, a través de procesos de formación integral, investigación y asesoría, se comprueba sin embargo que el tema de emprendimiento social y, más generalmente el de innovación social, hasta ahora no ha llamado tanto la atención de los académicos colombianos; la Universidad de los Andes es la única que propone programas formales y sustanciales al respecto. Se ve como una buena señal el hecho de que los profesionales de administración sean sensibilizados a los temas de ética en los negocios y a las problemáticas medio ambientales, pero no se puede sino comprobar el vacío existente en cuanto a la búsqueda y el estudio de formas de gestión y negocios innovadoras para responder a cuestiones sociales sin resolver. Fournier, A (2011).

En el ámbito local, con el gobierno japonés, a través de JICA (la Agencia de cooperación internacional) se está realizando el proyecto llamado OVOP (que traduce One Ville, One Product, una ciudad y un producto) en Tuchín (Córdoba), con el que se logró generar la denominación de origen del sombrero vueltiao, lo que significó un gran beneficio para la comunidad. Por otra parte, en Mompox, se están robusteciendo las organizaciones que laboran la filigrana; en el sector agropecuario se ha hecho la promoción y consolidación de modelos de circuitos económicos agroalimentarios, donde ha sido fundamental la participación de Agro-solidaria Riohacha. En la Costa se han conformado 40 micro-franquicias solidarias en Magangué, Sincelejo, Golfo de Morrosquillo, Montería, San Antero, Lorica y Gamarra. Allí están representadas nueve organizaciones con siete unidades productivas que han beneficiado a 226 familias. (El Tiempo, 2015); esta información nos muestra entonces el panorama del macro-entorno que enmarca nuestro proyecto de investigación. 
Por su parte hablar del contexto histórico del conflicto armado en Córdoba es relevante, ya que gracias a este los fenómenos del desempleo, la pobreza, los desplazamientos de campesinos a las zonas rurales han aunado en frenar el desarrollo económico de la región; desde mediados del siglo XX, el departamento de Córdoba, dada su ubicación estratégica, ha contado con la presencia de grupos armados que se disputan el control territorial de la región, pues posee una amplia salida al mar por el río Sinú y conecta la región Caribe con el interior del país; además se da la consolidación de grupos privados de seguridad que, a la postre, se convertirían en ejércitos contrainsurgentes como las Autodefensas Campesinas de Córdoba y Urabá (ACCU). CINEP (2016).

La forma en la que proliferó el latifundio y la figura del gran hacendado permitió a unas pocas familias, emparentadas con comerciantes e inmigrantes, acaparar miles de hectáreas de tierra, lo que les permitió no solo monopolizar la producción en todas sus escalas, sino también establecer determinadas formas de relacionamiento social y político; haciendo de Córdoba la plataforma del proyecto paramilitar debido a las estructuras de poder previamente consolidadas a través del modelo de hacienda, ya que, como se mencionó anteriormente, este incluía grupos de seguridad privada que monopolizaron el uso de la fuerza en el ámbito regional y establecieron dinámicas sociales y culturales en las que la defensa de la propiedad privada, el modelo ganadero y la extracción agroindustrial debía fomentarse incluso por medio de las armas (Aponte, 2014, p.146).

Todos estos problemas en el departamento de Córdoba han generado atraso y estancamiento de la economía, siendo Montería una de las ciudades con el PIB per cápita más bajo del país. Periódico El tiempo (Noviembre/2017).

Este análisis del contexto internacional, nacional y local, muestra que el apoyo a la investigación, grupos de estudio, desarrollo e innovación son cruciales, para el desarrollo y determinantes para la mejora continua de la economía regional, y más con respecto al tema del emprendimiento. Se quiere estudiar la prospectiva de emprendimiento en estudiantes de pregrado de la UCC sede Montería, ya que estos son los más susceptibles a tener dudas e interrogantes por resolver acerca de su desarrollo profesional y tienen una mente abierta a nuevas ideas que pueden ser la clave para su futuro y por ende para el futuro de córdoba y toda la región sinuana. 


\section{MÉTODOS}

El tipo de estudio que se llevó a cabo en esta investigación es descriptivo con un enfoque cuantitativo; ya que se busca identificar rasgos característicos de una determinada situación, evento o hecho; en este caso, se quieren conocer las características comunes de la población objeto, a través de encuestas dirigidas y el análisis estadístico de las mismas, el objeto estudiado son los estudiantes de la sede UCC Montería. Para llegar a darle respuesta, se intenta recolectar información, datos, detallar dimensiones, componentes y rasgos. Ferreyra \& De Longhi (2014).

Por su parte el enfoque cuantitativo es como lo explica Hernández, Fernández \& Baptista (2010); es el que usa la recolección de datos para probar hipótesis, con base en la medición numérica y el análisis estadístico, para establecer patrones de comportamiento y probar teorías; en esta oportunidad se diseñó una herramienta para recopilar información cuantitativa y hacer un análisis estadístico para llegar a una conclusión, y hacer una propuesta de mejora.

También se realiza investigación exploratoria; según Hernández \& Coello (2006) se realiza cuando existe una problemática y no se tiene una idea clara del asunto en cuestión, en este caso se necesita recopilar información para organizar el marco teórico de la investigación, el tema estudiado es el emprendimiento social, economía creativa o economía naranja, industria cultural; que son temas relativamente nuevos y por lo tanto se parte de la investigación exploratoria para recopilar la información necesaria y a partir de esta llegar a la investigación descriptiva.

La población total de los estudiantes de la sede de la universidad Cooperativa de Colombia sede Montería al año 2017 es 4.090 estudiantes según el DARC (2017); distribuidos en las facultades de Derecho, Ingeniería de Sistemas, Psicología y la Facultad de Ciencias Administrativas, Contables y Afines; de la cual se tomó una muestra de 350 estudiantes utilizando la fórmula para estimar la muestra en poblaciones finitas, con un nivel de confianza de $95 \%$ y una estimación de error del $5 \%$, que se puede apreciar en la tabla 1.

Luego de sacar este tamaño muestral se aplica el muestreo estratificado por programas, semestres y jornadas y luego el muestreo aleatorio simple por Excel; para determinar cuántas personas de cada programa realizarían la encuesta, teniendo en cuenta que todos tuvieran la misma probabilidad de selección. 
Tabla 1

MATRIZ DE CÁLCULO DE MUESTRA PARA POBLACIONES FINITAS

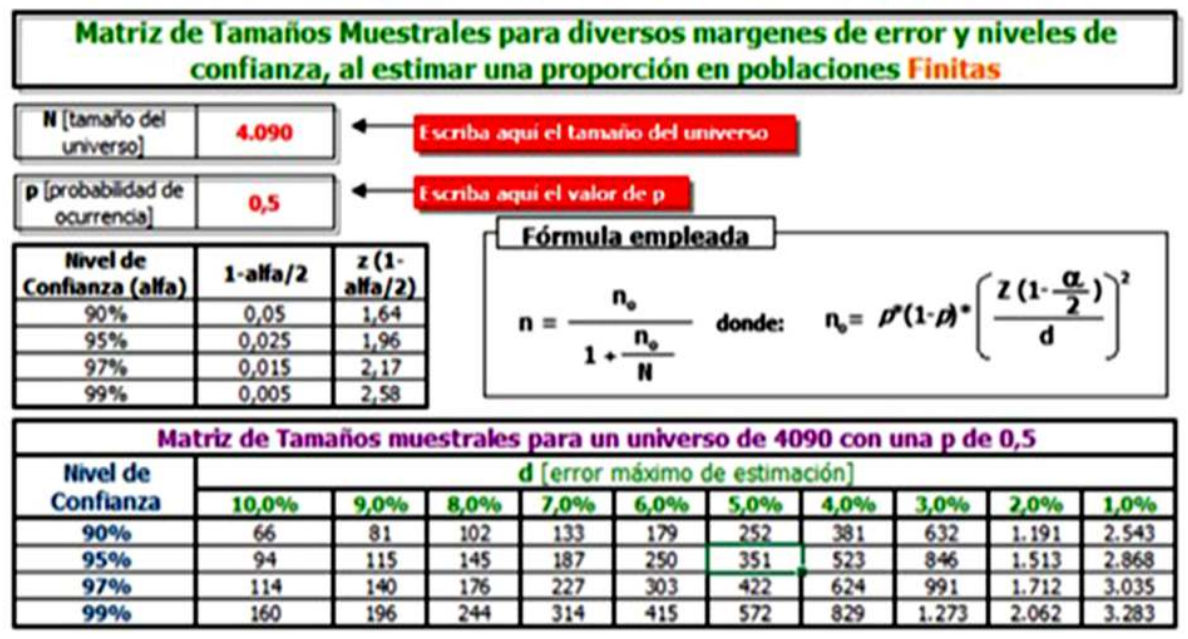

Tabla 2

MUESTREO ESTRATIFICADO POR PROGRAMAS

\begin{tabular}{|l|r|r|r|r|c|c|}
\hline \multicolumn{1}{|c|}{ PROGRAMA } & Diurno & Nocturno & \multicolumn{1}{c|}{ Total } & \multicolumn{1}{c|}{$\%$} & Muestra & Verificación \\
\hline Administración de Empresas & 145 & 78 & 223 & $6 \%$ & 20 & 20 \\
\hline Administración de Empresas C & 219 & 108 & 327 & $9 \%$ & 30 & 30 \\
\hline TOTAL ADMINISTRACIÓN & 364 & 186 & 550 & $14 \%$ & 50 & \\
\hline Contaduría Pública & 113 & 74 & 187 & $5 \%$ & 17 & 17 \\
\hline Contaduría Pública C & 377 & 175 & 552 & $14 \%$ & 50 & 50 \\
\hline TOTAL CONTADURIA PUBLICA & 490 & 249 & 739 & $19 \%$ & 67 & \\
\hline Derecho & 914 & 215 & 1129 & $29 \%$ & 103 & 103 \\
\hline Derecho C & 109 & 0 & 109 & $3 \%$ & 10 & 10 \\
\hline TOTAL DERECHO & 1023 & 215 & 1238 & $32 \%$ & 113 & \\
\hline Ingenieria de Sistemas & 37 & 0 & 37 & $1 \%$ & 3 & 3 \\
\hline Ingeniería de Sistemas C & 137 & 0 & 137 & $4 \%$ & 12 & 12 \\
\hline TOTAL INGENIERIA & 174 & 0 & 174 & $5 \%$ & 16 & \\
\hline Psicología & 867 & 269 & 1136 & $30 \%$ & 103 & 103 \\
\hline TOTAL GENERAL & 2.918 & 919 & 3.837 & $100 \%$ & 349 & 349 \\
\hline
\end{tabular}

Fuente: Elaboración propia. 
El formato de encuesta que se aplicó fue desarrollado basándose en otras investigaciones que han servido para grados de maestría; en este caso las preguntas de la sección I y III, se basaron en la investigación de Arbeláez, L (2011); titulado: "Emprendimiento social: factores críticos de éxito y fracaso para el mejoramiento del programa de generación de ingresos de la presidencia de la República de Colombia, dirigido a población en situación de desplazamiento por la violencia en Caldas". Por otra parte, se utilizaron las investigaciones de Llanos, J (2008); titulado: "Análisis de la oferta y demanda de servicios de apoyo para la creación de empresas: Caso Montería", y del artículo "El emprendimiento social en la formación integral" publicado por Ocampo, D (2016) de la universidad EAN.

Luego de elaborado el primer borrador de la herramienta de investigación, se procedió a aplicar una prueba piloto de 30 encuestas a los estudiantes de VI semestre del programa de Administración de empresas jornada diurna, y IV semestre del programa de Contaduría jornada diurna, con ayuda de los estudiantes pertenecientes al semillero cuya finalidad era establecer la claridad del formato y si había sugerencias por parte de los estudiantes.

Luego de esta prueba piloto se hicieron ajustes en cuanto a las instrucciones en el diligenciamiento de la encuesta con el fin de facilitar la comprensión y la secuencia en que debían contestar las preguntas. Por tanto, la herramienta definitiva que se aplicó, luego de la prueba piloto, quedó con la siguiente estructura:

1. Información general.

2. Información institucional.

3. Generación de la idea.

Las 3 partes anteriores suman un total de 35 preguntas, sin embargo, la numeración de las preguntas abarca las partes 2 y 3 para tener en total 27 preguntas. Las preguntas se diseñaron en formato de preguntas cerradas de selección múltiple con única respuesta y con múltiple respuesta, y solo se dejó una pregunta abierta con el fin de facilitar la tabulación de los resultados, que fue la pregunta final.

\section{RESULTADOS}

Dentro de los resultados encontrados, se quiso hacer énfasis en 6 preguntas relevantes las cuales proporcionaron la siguiente información: 


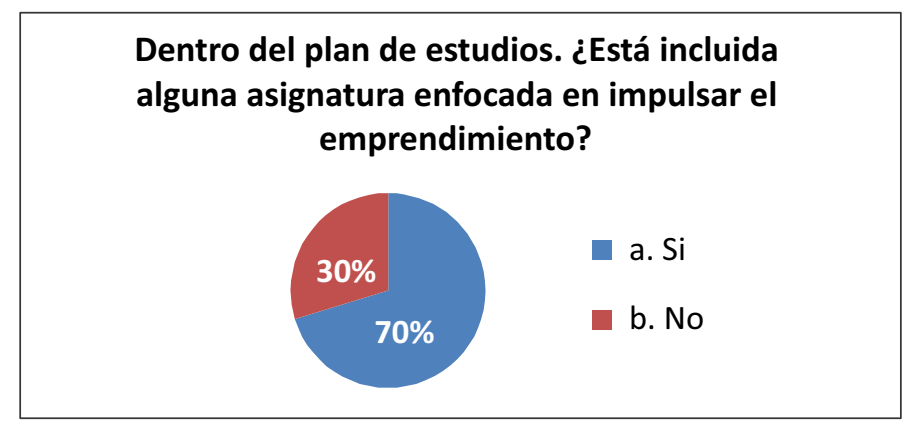

Gráfico 1. Pregunta I (Información institucional).

Fuente: Elaboración propia.

En términos generales la Universidad propende por impartir asignaturas enfocadas en promover el emprendimiento entre sus estudiantes, lo cual se ve reflejado en un $70 \%$ que afirma haber cursado una de estas asignaturas.

En el programa de Ingeniería de sistemas se encuentran las 3 electivas que se desarrollan en el plan de estudio y las cuales son: Electiva I (Ideación), Electiva II (Prototipado), y Electiva III (Planes de negocio).

En la Facultad de Ciencias Administrativas, Contables y afines, que agrupa a los programas de Administración de empresas y Contaduría pública, tiene las asignaturas Oportunidades de negocio y Plan de negocio.

En el programa de Psicología, los estudiantes cuentan con la asignatura Psicología organizacional, donde a lo largo del semestre hacen el montaje de una empresa con todos sus elementos, y al final del mismo hacen la presentación de la empresa donde muestran los productos o servicios de la misma en una feria con stands dentro del aula de clase.

En el programa de Derecho, no se tiene una asignatura enfocada en el emprendimiento como tal, los estudiantes cursan la asignatura Derecho comercial y de las sociedades, donde crean una empresa, pero dentro del concepto de empresa jurídica que trae el Código de comercio, pasando por todos los requerimientos y obligaciones de las personas naturales o jurídicas.

Es importante destacar que existen asignaturas transversales llamadas Institucionales, en donde se trabaja todo lo relacionado con el sector solidario del cual hace parte la Universidad, y específicamente se encuentra la asignatura Institucional III, enfocada en la temática de Emprendimiento solidario. Estas asignaturas son vistas por todos los estudiantes y son de modalidad virtual. 


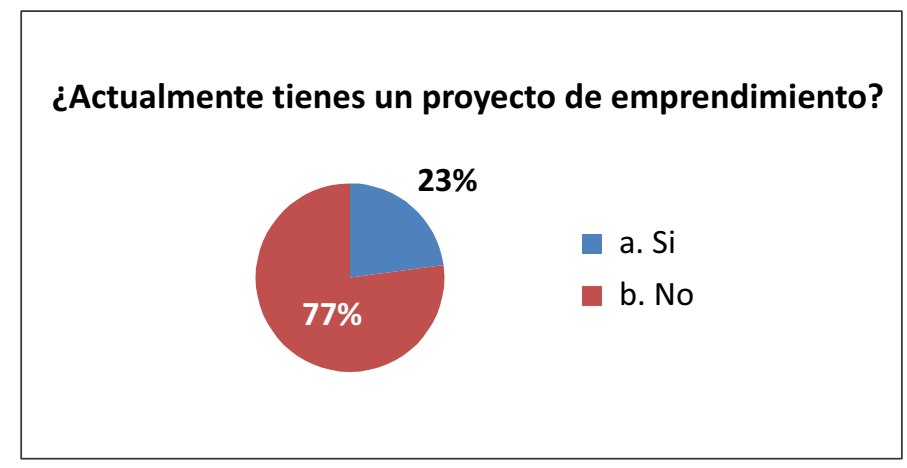

Gráfico 2. Pregunta 7 (Generación de idea).

Fuente: Elaboración propia.

Esta pregunta demuestra que a pesar de que la Universidad promueve el espíritu emprendedor como quedó plasmado en la pregunta sobre el plan de estudio, es preocupante observar que solo un número reducido de estudiantes han visto el emprender como opción para su proyecto de vida, esto deja la puerta abierta para el desarrollo de estrategias que busquen mejorar este índice y los estudiantes puedan ser generadores de proyectos productivos que impacten la región y el entorno del cual hacen parte.

El programa que tiene un mayor índice de estudiantes con proyectos de emprendimiento es ingeniería de sistemas, el cual a través de las 3 electivas que se desarrollan en el plan de estudio y las cuales son: Electiva I (Ideación), Electiva II (Prototipado), y Electiva III (Planes de negocio) que buscan incentivar en los estudiantes en su proceso emprendedor; dicho resultado coincide a lo afirmado por los estudiantes del programa de ingeniería los cuales respondieron afirmativamente al momento de indagar sobre asignaturas relacionadas con esta área dentro de su plan de estudio.

Y el programa que obtuvo los resultados más bajos fue el programa de Psicología, a pesar de contar con la asignatura Psicología organizacional, y que en ella presentan el proyecto de empresa desarrollado en todo el semestre, casi el $82 \%$ de los estudiantes encuestados manifestaron no tener ningún proyecto de emprendimiento en curso. 


\section{Sector de desarrollo del proyecto:}

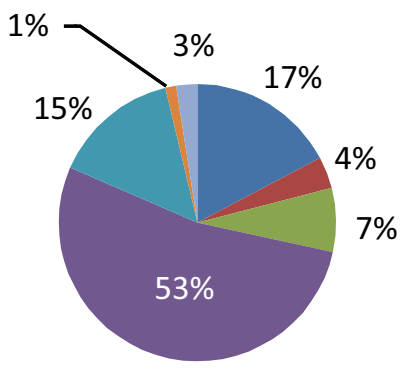

a. Social

b. Ambiental

c. Industrial

d. Comercial

e. Servicios

f. Tecnología

g. Otro

\section{Gráfico 3. Pregunta 8 (Generación de idea).}

Fuente: Elaboración propia.

Dentro del 23\% que marcaron que, sí tienen un proyecto de emprendimiento, se destacaron los siguientes sectores de desarrollo: social, comercial y servicios.

Es relevante mencionar que el Sector comercial es el predominante en los programas de Contaduría pública, Administración de Empresas, Derecho y Psicología. En cambio, el único programa que busca incentivar en sus estudiantes el emprendimiento social es Ingeniería de sistemas, lo cual se puede convertir en un referente para replicar a los demás programas de la Sede Montería. Esta situación nos arroja un interrogante sobre el iPor qué si el ADN de la Universidad Cooperativa de Colombia está enfocado en el sector solidario, solo uno de los 5 programas académicos ha logrado fortalecer este aspecto? Esto permite que se deba replantear la metodología y las estrategias que se están utilizando actualmente en las asignaturas enfocadas en la economía solidaria. 


\section{La idea de negocio fue creada por:}

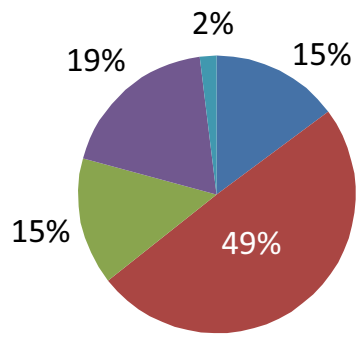

a. Necesidad

b. Deseo de emprender

c. Solucionar problema

d. Oportunidad

Gráfico 4. Pregunta 15 (Generación de idea).

Fuente: Elaboración propia.

Esta pregunta se planteó de múltiple respuesta. De las opciones planteadas, el deseo de emprender obtuvo el mayor porcentaje con un 50\%, de lo cual se deduce que la mayoría de los estudiantes contemplan en su proyecto de vida el ser independientes con su proyecto de negocio. En segundo lugar, se encuentra la identificación de una oportunidad con un 19\%, esto se relaciona con el deseo de emprender, donde los estudiantes siempre están en búsqueda de nuevas oportunidades que permitan materializar sus proyectos de negocios. Por otro lado, se resalta que, en el programa de Ingeniería de sistemas, prima el deseo de solucionar un problema como principal motivación para emprender, demostrando con esto la esencia misma del programa, el cual propende por la solución de problemas del entorno, característica particular de los que estudian esas áreas de formación. De estos resultados podemos inferir que es necesario que, dentro de estas asignaturas, se haga énfasis en la importancia de analizar el entorno con el fin de identificar problemas y oportunidades, los cuales son claves al momento de emprender. 


\section{¿Qué factores usted considera un limitante para crear empresa?}

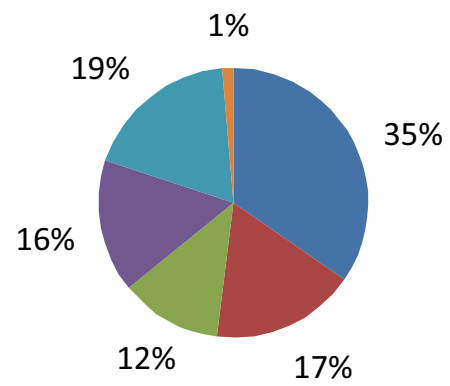

a. Falta de recursos

- b. Falta de capacitación

c. Falta de tiempo

d. Falta de apoyo

e. Falta de motivación

f. Otro

Gráfico 5. Pregunta 21 (Generación de idea).

Fuente: Elaboración propia.

Esta pregunta se planteó de múltiple respuesta. De las opciones planteadas, los estudiantes ven como principales limitantes a la hora de emprender, la falta de recursos (35\%), y la falta de motivación (19\%). Estas respuestas se pueden relacionar con el hecho de que la falta de recursos, conduce a la desmotivación. Sin embargo, cabe resaltar que dentro de estas 2 limitantes, la falta de recursos no debe mirarse como un obstáculo a la hora de materializar los proyectos, este aspecto debe resaltarse en las asignaturas que ven dentro de sus planes de estudio, las cuales deben socializar con los estudiantes las diferentes instituciones públicas y privadas que apoyan todas las iniciativas de emprendimiento, entre las que se encuentran: El Fondo Emprender del Sena, Propaís, el Concurso Venture, entidades financieras como Mundo Mujer, entre otras. De igual forma, se debe procurar aumentar el grado de motivación y acompañamiento para que esas ideas que tienen y el deseo de emprender no se vean frustrados por las razones anteriores. 


\section{¿Tienes en mente empezar alguna idea o proyecto de empresa?}

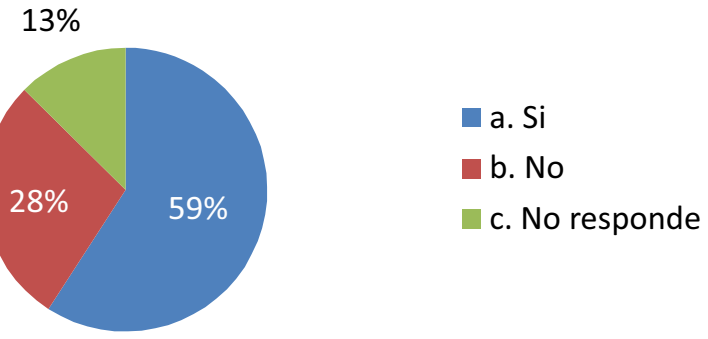

Gráfico 6. Pregunta 22 (Generación de idea). Fuente: Elaboración propia.

Del total de los estudiantes encuestados el 59\% manifestó su deseo de emprender su idea de negocio, lo que indica que se debe realizar un proceso arduo de sensibilización y motivación a la mitad de la población, teniendo como precedente que hubo un porcentaje que no respondió a esta pregunta, dejando la puerta abierta al deseo de emprender, y que en el momento de responder la encuesta no tenía una idea clara y por eso se dio esta respuesta.

En esta pregunta podemos concluir, que la falta de motivación para emprender, también puede verse relacionada con la falta de recursos que los estudiantes manifestaron como principal limitante, por lo tanto, retomamos que en estos aspectos es que debe reforzarse el acompañamiento por parte de la academia y de la universidad como institución formadora. 


\section{DISCUSIÓN}

a. La teoría analizada refleja que la Economía solidaria es la unión perfecta entre actividad económica y responsabilidad con las comunidades.

b. La Economía Solidaria genera riqueza, bienestar y calidad de vida en la población más excluida.

c. El emprendimiento social parte de la persona hacia la comunidad para satisfacer sus necesidades.

d. Fomentar el espíritu empresarial es una responsabilidad del estado con la academia de allí surge el semillero de proyectos productivos que luego se convierten en empresa.

e. La economía social y el emprendimiento social, deben unir fuerzas con entidades, tanto del estado como del sector privado, para favorecer la creación de un sistema social colaborativo y para la estructuración del sector mediante la creación de capital social.

f. La Universidad propende por impartir asignaturas enfocadas en promover el emprendimiento entre sus estudiantes; existen asignaturas transversales llamadas Institucionales, en donde se trabaja todo lo relacionado con el sector solidario del cual hace parte la Universidad, y específicamente se encuentra la asignatura Institucional III, enfocada en la temática de Emprendimiento solidario; estas asignaturas son vistas por todos los estudiantes y son de modalidad virtual.

g. A pesar que la Universidad promueve el espíritu emprendedor como quedó plasmado en la pregunta sobre el plan de estudio, es preocupante observar que solo un número reducido de estudiantes han visto el emprender como opción para su proyecto de vida.

h. El programa que tiene un mayor índice de estudiantes con proyectos de emprendimiento es ingeniería de sistemas y el que menos tiene, con casi el 82\% de los estudiantes encuestados, es Psicología.

i. Dentro del $23 \%$ que, sí tienen un proyecto de emprendimiento, se destacaron los siguientes sectores de desarrollo: social, comercial y servicios; es relevante mencionar que el Sector comercial es el predominante en los programas de Contaduría pública, Administración de Empresas, Derecho y Psicología. En cambio, el único programa que busca incentivar en sus estudiantes el emprendimiento social es Ingeniería de sistemas. 
j. A pesar que el ADN de la Universidad Cooperativa de Colombia está enfocado en el sector solidario, solo uno de los 5 programas académicos ha logrado fortalecer este aspecto.

k. Se deben replantear la metodología y las estrategias que se están utilizando actualmente en las asignaturas enfocadas en la economía solidaria.

1. Dentro del $23 \%$ que marcaron que sí tienen un proyecto de emprendimiento; se concluye que el deseo de emprender obtuvo el mayor porcentaje con un $50 \%$, de lo cual se deduce que la mayoría de los estudiantes contemplan en su proyecto de vida el ser independientes y en segundo lugar, se encuentra la identificación de una oportunidad con un 19\%, esto se relaciona con el deseo de emprender, donde los estudiantes siempre están en búsqueda de nuevas oportunidades que permitan materializar sus proyectos de negocios.

$\mathrm{m}$. Por otro lado, se resalta que, en el programa de Ingeniería de sistemas, prima el deseo de solucionar un problema como principal motivación para emprender, demostrando con esto la esencia misma del programa, el cual propende por la solución de problemas del entorno, característica particular de los que estudian esas áreas de formación.

n. De estos resultados podemos inferir que es necesario, dentro de estas asignaturas, hacer énfasis en la importancia de analizar el entorno con el fin de identificar problemas y oportunidades, los cuales son claves al momento de emprender.

o. Los estudiantes ven como principales limitantes a la hora de emprender, la falta de recursos (35\%), y la falta de motivación (19\%).

p. Del total de los estudiantes encuestados el 59\% manifestó su deseo de emprender, lo que indica que se debe realizar un proceso arduo de sensibilización y motivación a casi la mitad de la población que contestó negativamente. 


\section{REFERENCIAS BIBLIOGRÁFICAS}

Aguilar, C (2014). Elementos para la construcción del direccionamiento estratégico de Organizaciones Solidarias en Colombia durante el período 2014-2018: Una identificación desde el enfoque prospectivo. Documento técnico. Centro de investigaciones y prospectiva, Institución Universitaria ESUMER. Medellín.

Arana, A (2015). "Los recobros son el cáncer financiero del sistema de salud”. Gran foro sobre cooperativismo. La República. Recuperado de: http://www.larepublica.co/\%E2\%80\%9Closrecobros-son-el-c\%C3\%A1ncer-financiero-del-sistema-de-salud\%E2\%80\%9D_280541

Arbeláez, L (2011). "Emprendimiento social: factores críticos de éxito y fracaso para el mejoramiento del programa de generación de ingresos de la presidencia de la República de Colombia. Dirigido a población en situación de desplazamiento por la violencia en caldas. Trabajo para optar al título de maestría. Universidad Nacional de Colombia -Sede Manizales. Recuperado de: http://www.bdigital.unal.edu.co/3821/1/linamariaarbelaezrendon.2011.pdf

CINEP/Programa por la paz (2016). Tierra y Territorio en el Departamento de Córdoba en el escenario del postconflicto. $1^{\underline{a}}$ Ed. Casa Nabú Servicios Editoriales S.A.S; Equilibrio Gráfico Editorial Ltda. y Offset Gráfico Editores SA. Bogotá, Colombia.

DARC (2017). Población total de los estudiantes de la sede de la universidad Cooperativa de Colombia sede Montería, año 2017.

Ferreyra, A \& De Longhi, A (2014). Metodología de la investigación I. Córdoba. AR. Ed. Brujas. Recuperado de: ProQuest ebrary. 15 mayo 2017.

Fournier, A (2011). Documentos de Investigación. Emprendimiento Social. Universidad Central. Bogotá. Ed. Fundación Universidad Central.

González, F (2015). "Retos y Desafíos de la Prospectiva del Emprendimiento Social y Solidario de las ciudades del futuro". Sobre las empresas creadas por los egresados entre el año 2010 y 2014 de la Universidad Nacional Abierta y a Distancia UNAD, pertenecientes al Centro de Educación a Distancia CEAD. Sogamoso.

Hernández, Fernández \& Baptista (2010). Metodología de la Investigación. México. McGraw-Hill. 5 ed.

Hernández, R \& Coello, S (2006). "Método científico de investigación". El proceso de investigación científica. Madrid. Es. Editorial Universitaria. Recuperado de: ProQuest ebrary. 15 mayo 2017.

Llanos, J (2008). Análisis de la oferta y demanda de servicios de apoyo para la creación de empresas: Caso Montería. Proyecto para optar por el título de Magister MBA. Fundación Universidad del Norte. Barranquilla, Colombia.

Ocampo Eljaiek, D. (2016). El emprendimiento social en la formación integral. Revista Escuela de Administración de Negocios, (81), 175-190. Recuperado de:

http://www.redalyc.org/articulo.oa?id=20649705011

OIT \& COOP (2016). Las cooperativas y los Objetivos de Desarrollo Sostenible Debate sobre el desarrollo después de 2015. Recuperado de: http://www.ilo.org/wcmsp5/groups/public/... ed_emp/-.-emp_ent/-.-coop/documents/publication/wcms_307228.pdf 
Pallares, S (2011). Emprendimiento Creativo y Economía Social. Observatorio latinoamericano de gestión cultural. Recuperado de:

http://observatoriocultural.udgvirtual.udg.mx/repositorio/bitstream/handle/123456789/32

/Espinosa\%20\%20EMPRENDIMIENTO\%20CREATIVO\%20Y\%20ECONOM\%C3\%8D

A\%20SOCIAL\%20.pdf?sequence $=1$

Prada, J (2015). El debate de la creatividad y la economía en las ciudades actuales y el papel de los diferentes actores: algunas evidencias a partir del caso de estudio de Madrid. Scielo. n.87, pp.62-75. ISSN 2448-7279. http://dx.doi.org/10.14350/rig.40700.

Periódico El tiempo. El sector solidario, eje de la inclusión y tejido social. 27 de agosto 2015. Recuperado de: http://www.eltiempo.com/contenido-comercial/especiales-comerciales/elsector-solidario-eje-de-la-inclusion-y-tejido-social/16299379

Periódico el Tiempo (2017). Las 10 regiones que más aportan a la economía de Colombia. Recuperado de: https://www.eltiempo.com/colombia/otras-ciudades/las-regiones-masricas-del-pais-de-acuerdo-a-su-aporte-al-pib-146508

PNUD \& UNESCO, (2014). Informe sobre la economía creativa. Edición especial (2013). Ampliar los cauces del desarrollo local. Recuperado de: www.unesco.org/culture/pdf/creative economy-report-2013-es.pdf

Silveira, Y; Cabeza, D \& Fernández, V (2016). Emprendimiento: perspectiva cubana en la creación de empresas familiares. European Research on Management and Business Economics. Vol. $22(2)$; p.p. 70-77.

Zuluaga, C (2016). Cooperativismo y Postacuerdo: Con solidaridad la paz es sostenible. Organizaciones solidarias. 21.pp.14-17. Recuperado de:

http://www.orgsolidarias.gov.co/sites/default/files/archivos/Revista\%20Organizaciones\%20 Solidarias\%20Edici\%C3\%B3n\%2021\%20-\%202016.pdf 